\title{
THE NUMBER OF FORMED ELEMENTS IN THE URINARY SEDIMENT OF NORMAL INDIVIDUALS*
}

\author{
By T. ADDIS \\ (From the Department of Medicine, Stanford University Medical School, San Francisco),
}

(Received for publication February 23, 1926)

The general methods which for so long have been used in counting the cells of the blood are applicable in the enumeration of the formed elements of the urine. But in urine as opposed to blood there are such wide variations in volume, salt concentration and reaction that, it is only under certain special conditions that quantitative results can be obtained. Thus, in order to eliminate the effect of changes in volume the urine must be collected over a measured interval of time, so that the number of formed elements excreted per unit of time may be determined. Rates of excretion, not concentrations, are essential, for otherwise it would be impossible to tell whether a change from, say, 200 to 100 in the number of cells per cubic centimeter of urine was due to a decrease in the number of cells or to an increase in the volume of urine. Again, certain changes in salt concentration and reaction may lead to the partial or complete disappearance of some of the formed elements of the urine. In dilute and alkaline urine red blood. cells may be so completely lysed that they cannot be counted, and hyaline casts dissolve. A pronounced decrease in the number of casts in the urine of patients with Bright's disease may be readily brought about by simply increasing the volume of urine by giving water, since the hyaline casts and all casts with a hyaline matrix disappear when the urine becomes dilute. It has also been noted that a certain acidity, the degree of which depends on the salt concentration of the urine, is necessary for the preservation of hyaline casts. In test tube experiments it has been found that the minimal concentration of $\mathrm{NaCl}$ required to keep suspensions of washed hyaline casts from

* This work was aided by the Wellington Gregg Fund for the investigation of Bright's disease. 
dissolving increases as the $\mathrm{H}$-ion concentration is decreased. These more or less qualitative observations are sufficient to indicate that reliable counts can be secured only in collections of urine in which a certain degree of concentration and acidity is attained.

The particular means adopted to meet the requirements outlined above, the mode of urine collection and preservation, and the technique used in counting, have already been fully described elsewhere (1). In this paper are given the averages and ranges of variation in the twelve-hour rates of excretion of casts, red blood cells and of white cells and epithelial cells found in urine obtained from normal individuals.

The subjects were all working medical students who led a quite uniform and physically inactive existence. The collections were started at about 8:30 in the evening and the whole night urine was collected at one voiding twelve hours later when they came to work

TABLE 1

The rate of excretion per twelve hours of formed elements in the urine of normal men Averages from 82 counts on 74 individuals

\begin{tabular}{c|c|c}
\hline Casts & Red blood cells & White blood and epithelial cells \\
\hline 1,040 & 65,750 & 322,500 \\
\hline
\end{tabular}

in the morning. No fluids of any sort were taken after breakfast on the preceding day and as a consequence the twelve-hour volume was as a rule quite small. The average amount was $352 \mathrm{cc}$. The average specific gravity was 1.031 .

Table 1 gives the average number of casts, red blood cells, and white blood and epithelial cells found in 82 examinations of 74 individuals. In table 2 the counts are arranged in order of magnitude.

The average number of casts found in each twelve-hour collection was 1040. An inspection of table 2 shows that casts were found in 45 of the 74 individuals and were noted as absent in 29. But a consideration of the method employed makes it quite apparent that neither the figures given in those instances in which casts were discovered nor the zeros entered in the remainder can be regarded as having any individual quantitative significance. In the urine of patients with Bright's disease in which hundreds of thousands or millions of casts 
TABLE 2

Ranges of variation in the rates of excretion per twelve-hour night period of casts, red blood cells and white and epithelial cells in the urine of apparently healthy men

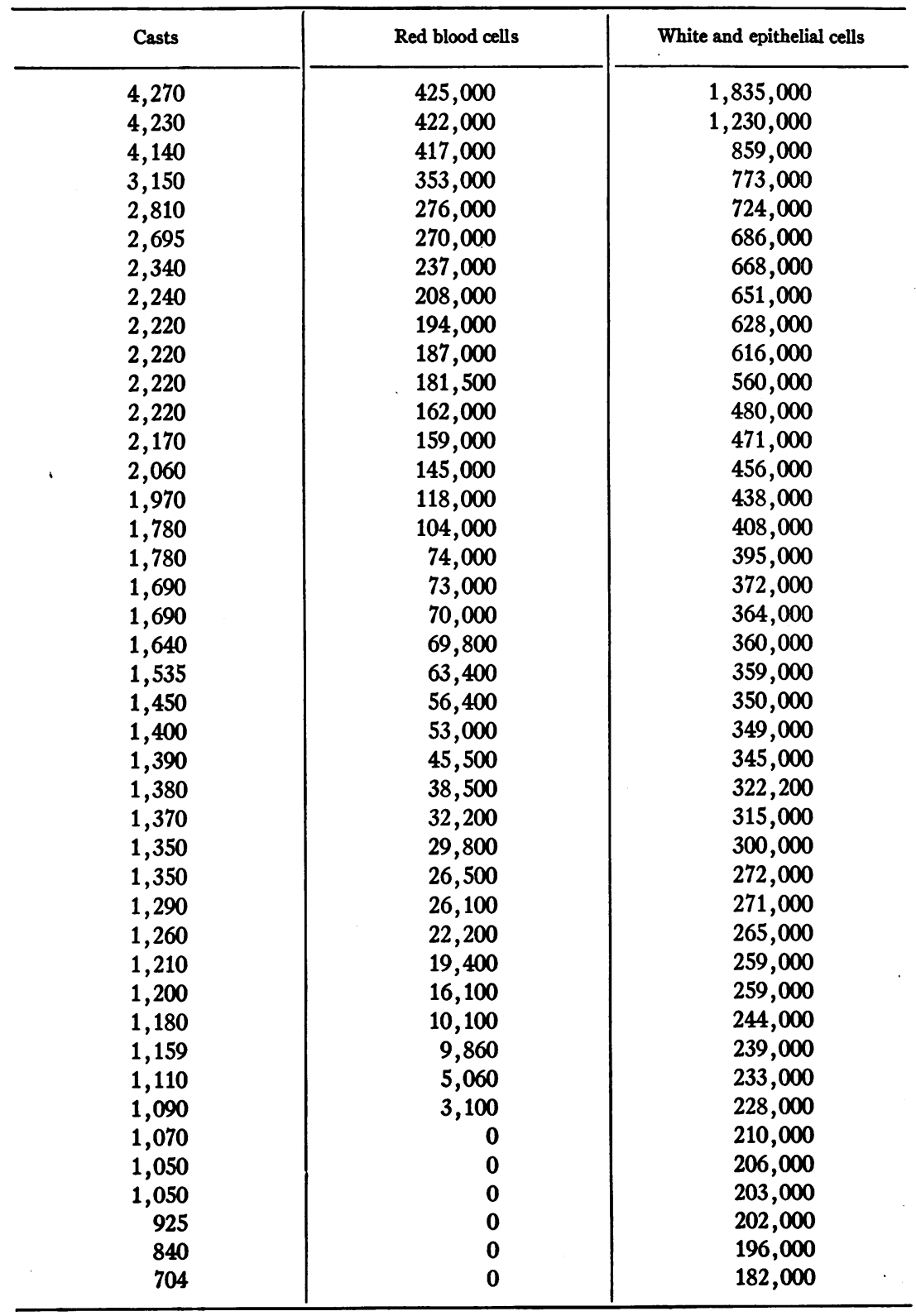


TABLE 2-Continued

\begin{tabular}{|c|c|c|}
\hline Casts & Red blood cells & White and epithelial cells \\
\hline $\begin{array}{r}676 \\
658 \\
645 \\
583 \\
580 \\
556 \\
528 \\
0 \\
0 \\
0 \\
0 \\
0 \\
0 \\
0 \\
0 \\
0 \\
0 \\
0 \\
0 \\
0 \\
0 \\
0 \\
0 \\
0 \\
0 \\
0 \\
0 \\
0 \\
0 \\
0 \\
0\end{array}$ & $\begin{array}{l}0 \\
0 \\
0 \\
0 \\
0 \\
0 \\
0 \\
0 \\
0 \\
0 \\
0 \\
0 \\
0 \\
0 \\
0 \\
0 \\
0 \\
0 \\
0 \\
0 \\
0 \\
0 \\
0 \\
0\end{array}$ & $\begin{array}{r}174,000 \\
172,000 \\
168,000 \\
165,000 \\
163,000 \\
158,000 \\
157,000 \\
147,000 \\
142,000 \\
139,000 \\
130,000 \\
130,000 \\
129,000 \\
126,000 \\
120,000 \\
119,000 \\
116,300 \\
113,400 \\
111,000 \\
90,800 \\
84,000 \\
80,400 \\
66,800 \\
60,600 \\
56,400 \\
40,000 \\
32,400\end{array}$ \\
\hline
\end{tabular}

are excreted every twelve hours the numerical results are trustworthy, but in these normal urines never more than 5 and often only 1 cast was seen in any one collection. It therefore seems quite likely that a more laborious search would have disclosed some casts in most if not in all of those specimens which are reported here as containing none. ${ }^{1}$ But though no reliance can be placed on any single figure of

${ }^{1}$ The total ruled area of 9 sq. $\mathrm{mm}$. in the ordinary type of blood counting chamber was counted over from 4 to 10 times with separate drops of sediment. In the majority of cases 8 drops were used. This represents a volume of 0.0072 
the series, the average result is probably a close approximation to the number of casts excreted by normal individuals when the conditions permit the formation and preservation of these structures.

The range of variation was from none to 4270 casts in twelve hours, and though here again no stress can be laid on the actually observed limits, they at least define the order of magnitude in the number of casts to be expected in the urine from normal individuals, and I have found this estimate of value in determining the significance of the sediment results in patients in whom some very minor renal lesion was suspected. It was of course for this reason that counts were made. The fact that urine from healthy individuals may contain casts was established by Haines and Skinner in 1898 (2).

The type of cast found was the hyaline variety. There were only three instances in which a single epithelial or granular cast was discovered. The hyaline casts only rarely contained any cell inclusions and fat droplets were never seen.

The average number of red blood cells was 65,700 per twelve-hour period. It seems to be generally assumed that the presence of red cells in urine is always indicative of some abnormality in the urinary tract, but this view is certainly erroneous. On the other hand they are probably not constant urinary constituents for I have sometimes failed to find them in spite of prolonged search in normal individuals in whose urine they had previously been present. They were discovered in 40 of the 64 examinations made, and might very well have been found in a larger percentage if more time had been spent in searching for them. However the main interest lay in the determination of the range of variation. This was found to extend from none to 425,000 per twelve-hour period, a fact of special importance in the diagnosis of the latent stage of haemorrhagic nephritis. It is clear from the above figures that it is only when the red blood cells are excreted at a rate of a million or more in each twelve hour period that they can be regarded as significant. However it should be remembered that without some experience and a good deal of care

cc. The sediment was concentrated by centrifuging from a $10 \mathrm{cc}$. sample of the mixed urine to a volume which varied from 0.1 to $0.3 \mathrm{cc}$. but usually $0.2 \mathrm{cc}$. With a count through $0.0072 \mathrm{cc}$. from a sediment of $0.2 \mathrm{cc}$. and a urine volume of $352 \mathrm{cc}$., the fraction of the total urine actually examined represents only 1 part in $\mathbf{9 7 2}$. 
one is apt to pass over many of the forms which red blood cells may assume in urine. Particularly in the urine of patients they may be fragmented or almost wholly lysed, so that only faint shadows remain which are easy to miss.

The average number of white blood cells and of small round epithelial cells in the twelve hour out-put of urine was 322,500 and the range of variation lay between $1,835,000$ and 32,400 . Both white blood and epithelial cells were counted together because it was frequently impossible to decide to which of these two classes a given cell belonged. Of course, from the standpoint of renal diagnosis, what is really wanted is a method by which those cells which come from the kidney can be distinguished from all others. A great many stains were tried but without success and in the end it was concluded that size was the only criterion. The large cells from the surface layers of the mucous membrane of the urinary tract were not included and the medium sized cells which originate from deeper layers were passed over when it was considered that their diameter was greater than that which could well be attained by even a swollen renal cell. This is admittedly a vague method of differentiation and one under which a number of cells from the prostate and other glands of the urinary tract are included. The wide range of variation in the counts is indeed in all probability to be accounted for largely by the inclusion of latent cases of prostatitis and urethritis. In practise however it has not proved an unsatisfactory standard because the number of white blood and epithelial cells found in the urine of patients in whom an active renal lesion exists is great enough to far surpass our upper limits of normality.

\section{CONCLUSIONS}

The rate of excretion of casts, red blood cells, and white blood and epithelial cells was determined in 74 medical students under conditions favorable to the preservation of these urinary constituents. The following results were obtained: 
Rate of excretion per twelve-hour period

\begin{tabular}{|c|c|c|c|}
\hline & Average & Lowest & Highest \\
\hline Casts. & 1,040 & 0 & 4,270 \\
\hline Red blood cells........... & 65,750 & 0 & 425,000 \\
\hline White blood and epithelial cells..... & 322,500 & 32,400 & $1,835,000$ \\
\hline
\end{tabular}

\section{BIBLIOGRAPHY}

1. Addis: Jour. Amer. Med. Assoc., 1925, lxxxv, 163.

2. Haines and Skinner: Jour. Amer. Med. Assoc., 1898, xxx, 234. 